B. VANTIJN

\title{
WERNER BLUMENBERG
}

Wenige Wochen nach dem plötzlichen Ableben seines Direktors, Professor A. J. C. Rüter, hat das Internationale Institut für Sozialgeschichte am I. Oktober 1965 mit dem Tode Werner Blumenbergs, des Leiters der Deutschland-Abteilung, einen zweiten schweren Verlust erlitten.

Kurz nach Kriegsende, sobald die Möglichkeit der Wiederaufrichtung des Instituts vorhanden war, hat Blumenberg diese Stellung beim Institut angetreten. Schon in den Jahren vor I940 hatte er das Institut kennengelernt. Als Emigrant in Amsterdam lebend hatte er seine allzu reichlich bemessene freie Zeit dazu verwendet, seine nie $\mathrm{zu}$ stillende Wissensbegierde zu befriedigen. Als Besucher war er mit den Zielsetzungen und im allgemeinen auch mit den damaligen Beständen bekannt geworden. Sein Gefühl der Verbundenheit mit dieser Institution war in jener Zeit eines unsicheren Emigrantendaseins erwacht und gefestigt.

Es ist aber wohl kaum anzunehmen, dass Blumenberg beim Antritt dieser neuen Tätigkeit auch nur geahnt hat, dass diese 20 Jahre lang, bis zu seinem Lebensende dauern würde. Denn eine gradlinige Fortsetzung seiner ehemaligen Beschäftigung war sie keinesfalls. Noch in seinen letzten Lebensjahren hat er sich mir gegenüber mit Nachdruck dahin geäussert, dass er doch eigentlich Politiker sei und dies seine Haltung hinsichtlich seiner Arbeit bestimme. Anfangs, daran kann nicht gezweifelt werden, hat er wohl fest damit gerechtnet, dass er bald wieder in der Politik seines Heimatlandes mitmachen könne. Noch mehrere Jahre hindurch hat er den Gedanken gehegt, zu politischer Tätigkeit zurückgehen zu können.

Allmählich, unmerklich, nachdem so viele der Bestände des Instituts in den Jahren des Wiederaufbaus buchstäblich durch seine Hände gegangen waren, hatte sich in ihm eine Änderung vollzogen. Er wollte bei diesen Papieren bleiben, sich damit intensiver noch beschäftigen, sie zugänglich machen, selbst darin immer tiefer eindringen um Kenntnisse zu erweitern und Probleme zu klären. Das Gefühl, damals wohl noch nicht ins Bewusstsein getreten, dass die Arbeit im 
Institut gewissermassen doch auch eine Fortsetzung der früheren sei und der Politik, in dem hohen Sinne wie er sie verstand, förderlich, wird hier wohl mitbestimmend gewesen sein.

Es wäre somit falsch, hier von einem vollständigen Bruch zu reden. Vergegenwärtigt man sich wie Blumenberg zur politischen Journalistik und zur praktischen politischen Tätigkeit gestossen war, dann wird die persönliche Komponente der späteren Wahl auch deutlich.

Der am 2. . Dezember I900 in Hülsede geborene Sohn eines evangelischen Pfarrers, hatte auch selbst das Studium der Theologie - nebst der Orientalistik - gewählt, dem er in Göttingen und Marburg oblag. Diese Wahl darf nicht der Familientradition zugeschrieben werden. Sie war - es geht aus einigen Notizen und literarischen Versuchen aus jungen Jahren klar hervor - eine durchaus persönliche Entscheidung, begründet durch eigene Auffassungen und eigene Zielsetzungen. Im Amte sah er die Möglichkeit daran mitzuarbeiten, die Menschen und die Welt zu verbessern. Bezeichnenderweise befasst der Theologiestudent sich nebenher gründlich mit der Philosophie, wohl aus dem Bedürfnis heraus, auch für den späteren Historiker charakteristisch, die ganze Problematik in den breitesten Rahmen zu setzen und Gedanken und Vorstellungen auf ihren wesentlichen Wert zu prüfen.

Es war auch folgerichtig, dass er, zur Arbeit an und mit Mitmenschen sich berufen fühlend, sich nicht auf das Studium beschränkte, sondern ein praktisches Tätigkeitsfeld suchte innerhalb und ausserhalb der Studentenwelt. So war er schon Mitbegründer der demokratischen Studentenvereinigung und trat nach dem Kapp-Putsch der sozialdemokratischen Partei bei.

Pastor ist er dann nicht geworden. Als er anlässlich einer Probepredigt aus einem ihm zugetanen Munde zu hören bekam, dass dies eigentlich Philosophie sei, mag er sich wohl darüber klar geworden sein, dass hier seine Berufung nicht lag.

Eine Zeit lang, in schlimmster Inflationszeit, arbeitete er dann in einem Bergwerk. Es mag sein, dass er in dieser Weise das schwere Leben der Bergleute aus eigener Erfahrung kennenlernen wollte, es mag auch sein, dass er solchermassen seine vorbehaltlose Identifizierung mit dem Volk, mit der Arbeiterklasse hat bekunden wollen.

Nach diesem Erlebnis war er in den Jahren 1924 bis 1933 Redakteur an den sozialdemokratischen Zeitungen in Göttingen und Hannover. In dieser Zeit trat er auch vielfach als politischer Redner auf und hielt Rundfunkvorträge im Rahmen der sozialistischen Feierstunde.

Mit dem Herannahen des grossen Unheils brach für ihn die Periode praktischer politischer Tätigkeit an, einer ungewöhnlichen mühevollen und gefährlichen politischen Tätigkeit. Schon im Sommer 1932 
gründete er in Hannover insgeheim eine Gruppe, die sich auf die zu erwartende illegale Arbeit vorbereitete. Dies geschah „Auf Anlass des Parteivorstandes der SPD". Nur an vereinzelten Orten kam es zur Gründung einer derartigen Gruppe. Es ist bezeichnend, dass Blumenberg, der zurückhaltende, der irenische Mensch zu den Ausnahmen gehörte, die diesem Winke folgeleisteten. Er, der Leiter der Gruppe, hat auch diese Arbeit gewissenhaft und gründlich geleistet. Mit grosser Erfindungsgabe, gestützt auf ein ausgedehntes Studium von polizeilichen und illegalen Techniken und mit Gefahr für Freiheit und Leben, hat er schwierige Aufgaben, darunter Reisen ins Ausland, auf sich genommen. Er gehörte zu jenen bescheidenen schüchternen Menschen die, wenn es darauf ankommt in ihrem unbeirrbaren Bewusstsein der Verantwortlichkeit den Menschen und ihren eigenen Idealen gegenüber den erforderlichen Mut finden und das Schwierigste leisten, nicht im Rausche, sondern in voller Kenntnis der Gefahren.

Nachdem Blumenberg gerade noch rechtzeitig vor der geplanten Verhaftung, im Sommer 1936, nach Holland entkommen konnte, hat er im Spätherbst desselben Jahres über die Tätigkeit der Gruppe ein Memorandum abgefasst, das 1956 auszugsweise von Erich Matthias veröffentlicht worden ist. ${ }^{1}$ Dieses Memorandum, von eminenter Bedeutung für die Geistesgeschichte der deutschen sozialistischen Illegalität, gibt uns auch Auskunft über die damaligen politischen und historischen Ansichten seines Verfassers. Es geht daraus klar hervor, dass die Gruppe, auf jeden Fall ihr Leiter, sich der Tatsache bewusst war, dass man ganz andere Wege ging als die Sozialdemokratie in den vorhergegangenen Perioden. Aber man verspürte doch das Bedürfnis, die Tatsache, dass man anscheinend nicht aus dem gedanklichen Bemühen und den Erfahrungen früherer Zeiten lernen wollte, wo nicht zu entschuldigen, so doch zu rechtfertigen. Daraus kann der Satz verstanden werden: „Es gab ja keine sachliche Situation in der Vergangenheit, in der die jetzt aktuellen Fragen bereits einmal hätten durchdacht werden können."2

Aber darüber hinaus verspürt der Leser des Memorandums an manchen Stellen deutlich, dass der Verfasser in sich das weitere Bedürfnis empfindet, das Auftreten der Gruppe als in die Geschichte hineingestellt zu erfahren. Es war von der bisher eingehaltenen Linie scharf abgewichen worden. Es war eine bestimmte fatalistische Interpretation der Geschichte eingetauscht worden gegen eine aktivistische

1 Erfahrungen in der illegalen Arbeit (Auszug aus einem Memorandum vom November I936, hrsg. von E. Matthias), in: Vierteljahrshefte für Zeitgeschichte, IV (I956), S. 20I226.

2 Ebenda, S. 202. 
Auffassung der Politik, die Grundlage und Rechtfertigung des eigenen, manchmal improvisierenden, immer gefährlichen Vorgehens sein sollte. Über diese Tatsache war sich der Verfasser völlig im klaren. Trotzdem wollte er für sich die Sicherheit behalten oder gewinnen, dass er bei der Arbeit der Geschichte einen grossen, in ruhigeren Zeiten näher zu erklärenden Wert zuerkannt hatte.

Nachdem er dann während der Jahre bis 1945 , soweit dies in misslichen Verhältnissen möglich war - in der Besatzungszeit lebte er illegal, unterhielt aber trotzdem Verbindungen mit der Widerstandsbewegung -, seine vielseitigen grossen Kenntnisse noch erweitert hatte, konnte er, wie schon erwähnt, nach Kriegsende die Arbeit beim Institut anfangen. Wie er in der Arbeit und durch die Arbeit immer mehr mit dem Institut verwuchs, ist schon ausgeführt worden. Hier muss darauf hingewiesen werden, dass diese Arbeit in der ersten Zeit ebenso ermüdend, wie dem Anschein nach ganz subalterner Art war. Nach und nach kehrten die abtransportierten Bestände in das ausgeplünderte Institut zurück. Sie mussten in Empfang genommen, ausgepackt, vorläufig gesichtet, in einem späteren Stadium systematisch geordnet werden.

Mit offenen Augen, mit wachem Interesse hat Blumenberg sich dieser auch körperlich anstrengenden Arbeit entledigt. Er sah alles, behielt alles im Gedächtnis und wurde so wohl der beste Kenner der Bestände des Instituts. Er hatte nicht nur das detaillierte Inventar der eigenen, unvergleichlich reichhaltigen Abteilung fest im Kopfe, lange bevor diese beschrieben werden konnten, er kannte sich auch gründlich aus in den Besitztümern der anderen Abteilungen. Hatte er sich im Anfang mit der eben erwähnten bescheidenen Arbeit abfinden müssen, abfinden wollen, nachdem mehr oder weniger Ordnung geschaffen war, sah er sich mit anderen, gleichfalls dienenden Tätigkeiten überbürdet. Noch ehe die Sammlungen ganz geordnet waren, kamen schon die Besucher, die Dokumente studieren wollten. Blumenberg hat ihnen den Weg gewiesen, aber nicht nur den Weg durch die teilweise ungeordneten Papiere und die reichhaltigen periodischen Veröffentlichungen. Sondern er hat jüngeren Anfängern und auch älteren Fachleuten den wissenschaftlichen Weg, den sie zu gehen hatten, erleichtert.

Denn aus dem Politiker mit historischem Bewusstsein und historischen Kenntnissen hatte sich in den Jahren der Emigration und der Arbeit beim Institut nicht nur ein - theoretischer - Politiker mit echter historischer Bildung und historischem Weitblick entwickelt, sondern auch ein Historiker mit wissenschaftlichem Gewissen, der über wirklich ausgedehnte politische Bildung und Erfahrung ver- 
fügte. Dieser Historiker konnte lehren wie die Dokumente bearbeitet werden sollten, damit nicht nur saubere, sondern auch bedeutende Arbeit geleistet werde.

Seine Hilfsbereitschaft, seine Bescheidenheit, die wohl mitunter die Selbstverleugnung streifte, sind nur zu oft missbraucht worden. Dass soll hier einmal ganz offen ausgesprochen werden. Blumenberg konnte nicht nein sagen. Und so hat er immer wieder seine Zeit darauf verwendet, für Andere Untersuchungen durchzuführen, die sie selbst hätten vornehmen können.

Dabei drängte es ihn nach eigenen Veröffentlichungen. Er kannte die Möglichkeiten, die im Institut für die Bearbeitung der Themen, die ihn reizten, vorhanden waren. Er fühlte sich, wie er mir einmal sagte, „vollgesogen mit Tatsachenkenntnissen wie ein Schwamm". Auf Grund seiner Fähigkeiten und dieser Kenntnisse hat er dann grossen, oft massgeblichen Einfluss auf die Aufstellung der herausgeberischen Programme des Instituts gehabt.

Die eigenen Veröffentlichungen aber sind in den soer Jahren fast nut auf einige vereinzelte kleine wertvolle Arbeiten für das Bulletin of the International Institute of Social History beschränkt geblieben, denen sich dann in seinen letzten Lebensjahren noch einige für die International Review of Social History angeschlossen haben. Dokumentenveröffentlichungen mit beispielhaftem unbedingt unentbehrlichem und wirklich aufklärendem Kommentar und auf Dokumenten basierten Aufsätzen.

Daneben hat er dann im Bulletin 1950, Nr. 3 sein Inventar der „Zeitungen und Zeitschriften in der deutschen Sprache aus der Zeit von 1830 bis 1890 im Internationalen Institut für Sozialgeschichte" veröffentlichen können, ein wichtiges Hilfsmittel für Studierende auf diesem Gebiet, dem sich dann zehn Jahre später die grosse auf Vollständigkeit angelegte Veröffentlichung „Karl Kautskys literarisches Werk" anschloss. Auch diese als Ehrenbezeigung an den bedeutenden sozialistischen Theoretiker unternommene Arbeit ist an erster Stelle Hilfsmittel für das Studium Anderer.

Auf Verlegerinitiative gingen dann die beiden Veröffentlichungen zurück: „Kämpfer für die Freiheit” (J. H. W. Dietz Nachf., Berlin und Hannover 1958) und „Karl Marx in Selbstzeugnissen und Bilddokumenten" (Rowohlt, Reinbek bei Hamburg I962). Gewissermassen wird hier wieder angeknüpft bei der Bildungsarbeit, die der Autor vor I933 geleistet hatte. Der Gedanke, dass es für die sozialistische Bewegung gut, ja notwendig sei, sich auch in die Vergangenheit zu versenken, war bei der Abfassung dieser Bücher die eigentlich treibende Kraft. Es war dieser Gedanke, an den er auch in so anders gearteter aktivistischer Zeit festgehalten hatte, und den er 
denn auch in dem Memorandum von 1936 unzweideutig ausgesprochen hatte. Er war auch das wesentliche Motiv bei seiner ganzen Arbeit im Institut, der ordnenden, herausgeberischen und dem Studium Anderer förderlichen Arbeit.

Diese beiden „populären” Veröffentlichungen liegen auf dem Gebiet seines eigentlichen Studiums, vorzüglich das Marx-Buch. Sie zeichnen sich dann auch aus durch gründliche Sachkenntnis. Darüber hinaus kann festgestellt werden, dass der Autor weder das Verlangen hatte, die Probleme in unverantworteter Weise zu vereinfachen, noch Handlungen oder Motive zu beschönigen.

Das Ziel der sozialistischen Geschichtsschreibung wird im Vorwort seiner „Kämpfer” folgendermassen formuliert: „Gewiss hat eine politische Bewegung nicht das Bedürfnis, sich an Helden- und Heiligengeschichten zu erbauen. Es ist auch nicht förderlich, sich mit der Vergangenheit zu beschäftigen, um Analogien zu finden: um dann genau so zu entscheiden wie früher einmal. Aber wohl kann der Rückblick auf unsere Vergangenheit uns zeigen, wie wir geworden sind. Wir erkennen daraus unsere grossen Möglichkeiten und auch deren Grenzen."

Der Historiker Blumenberg kannte keinerlei schwächliche Ehrfurcht, weder vor den überlieferten Meinungen über Persönlichkeiten, noch vor einer - Bequemlichkeit in Wort und Tat fördernden - traditionellen Interpretation des früheren Geschehens. So hat er es verstanden, in seinem kleinen glänzend komponierten und inhaltsreichen Marx-Buch nach so vielen Marx-Biographien neue, das Wesentliche treffende Züge dem Bilde hinzuzufügen. Hier erscheint Marx nicht als der Mensch ohne menschliche Fehler und politische Unzulänglichkeiten, nicht als der Mensch, der immer genau den richtigen Weg kannte, sondern der immer bestrebt war, ihn angestrengt zu finden.

Von den grösseren Dokumentensammlungen, die Blumenberg geplant hatte, hat er nur August Bebels Briefwechsel mit Friedrich Engels noch fertigstellen können. ${ }^{1}$

Im Vorwort dieser Arbeit zeigt er den ganzen Umfang seines Könnens. Der Kenner ungezählter Dokumente - nicht nur der hier veröffentlichten - weiss, welche Legenden es zu zerstören gibt. Er ist imstande, mit seiner durch breit angelegtes Geschichtsstudium und politische Tätigkeit vertieften Einsicht den Dokumenten die richtigen Fragen zu stellen. Aus der Fülle seines Wissens konnte er Hinweise auf die verschiedensten Quellen geben, die für die weitere

1 August Bebels Briefwechsel mit Friedrich Engels, hrsg. von W. Blumenberg [Quellen und Untersuchungen zur Geschichte der deutschen und österreichischen Arbeiterbewegung, Bd. VI], Mouton \& Co., Den Haag 1965. 
Bearbeitung der angerührten Probleme von Bedeutung sind. Es geht aus dieser Arbeit klarer noch, als aus den vorangehenden hervor, wie sehr ihn das Problem des Verhältnisses von Theorie und Praxis fesselte. Er wusste, wie Jahrzehnte hindurch die Auffassung vorherrschend gewesen war, nach der die Theorie in jedem Moment fix und fertige Antworten für das politische Handeln verschaffen könne. Es war ihm durch Erfahrung und Nachdenken bewusst, wie diese Auffassung nicht nur zu Bequemlichkeit verführt hatte, sondern auch zur Heuchelei eines Auftretens ohne jede theoretische Überlegung, aber unter Bezugnahme auf theoretische Formeln. Andererseits fürchtete er, dass in der heutigen Zeit eine Theorie der Theorielosigkeit die Oberhand gewinnen könne.

So hatte er geplant, in einer grösseren Arbeit das Problem des Verhältnisses von Theorie und Praxis bei Karl Marx zu untersuchen. Es mag ihm dabei, aus früheren Arbeiten zu schliessen, wohl der Plan vor Augen gestanden haben zu zeigen, wie bei Marx jedenfalls immer die Absicht vorhanden war, zu jeder Zeit die Theorie nur zu verwenden zu einer genauen Analyse der jeweiligen konkreten Situation nach der sachlichen und der persönlichen Seite, und inwieweit und unter welchen Umständen er diesem Vorhaben untreu geworden ist.

Diese Arbeit hat Blumenberg nicht einmal mehr anfangen können. Eine Edition von Dokumenten und Korrespondenzen aus den soer Jahren des vorigen Jahrhunderts aus dem Kreise um Marx und Engels, hat er nicht mehr vollenden können. Müssig ist es, bei der Frage zu verweilen, was aus diesen Plänen und Ansätzen hätte werden können.

Ein gelehrter und weiser Mensch, dem Kollegen und viele ältere und jüngere Historiker wertvolle Anregung verdanken und nicht nur auf wissenschaftlichem Gebiet, ein hilfsbereiter gütiger und in seiner Bescheidenheit überlegener Mensch - so wird Blumenberg in der Erinnerung fortleben. 